\title{
Biovar-related differences apparent in the flea foregut colonization phenotype of distinct Yersinia pestis strains do not impact transmission efficiency
}

\author{
Athena Lemon, Janelle Sagawa, Kameron Gravelle and Viveka Vadyvaloo*
}

\begin{abstract}
Background: Yersinia pestis is the flea-transmitted etiological agent of bubonic plague. Sylvatic plague consists of complex tripartite interactions between diverse flea and wild rodent species, and pathogen strains. Transmission by flea bite occurs primarily by the Y. pestis biofilm-mediated foregut blockage and regurgitation mechanism, which has been largely detailed by studies in the model interaction between Y. pestis KIM6+ and Xenopsylla cheopis. Here, we test if pathogen-specific traits influence this interaction by determining the dynamics of foregut blockage development in X. cheopis fleas among extant avirulent PCD1-Y. pestis strains, KIM6+ and CO92, belonging to distinct biovars, and a non-circulating mutant CO92 strain (CO92gly), restored for glycerol fermentation; a key biochemical difference between the two biovars.
\end{abstract}

Methods: Separate flea cohorts infected with distinct strains were evaluated for (i) blockage development, bacterial burdens and flea foregut blockage pathology, and (ii) for the number of bacteria transmitted by regurgitation during membrane feeding. Strain burdens per flea was determined for fleas co-infected with CO92 and KIM6+ strains at a ratio of 1:1.

Results: Strains KIM6+ and CO92 developed foregut blockage at similar rates and peak temporal incidences, but the CO92gly strain showed significantly greater blockage rates that peak earlier post-infection. The KIM6+ strain, however, exhibited a distinctive foregut pathology wherein bacterial colonization extended the length of the esophagus up to the feeding mouthparts in $~ 65 \%$ of blocked fleas; in contrast to $32 \%$ and $26 \%$, respectively, in fleas blocked with CO92 and CO92gly. The proximity of KIM6+ to the flea mouthparts in blocked fleas did not result in higher regurgitative transmission efficiencies as all strains transmitted variable numbers of Y. pestis, albeit slightly lower for CO92gly. During competitive co-infection, strains KIM6+ and CO92 were equally fit maintaining equivalent infection proportions in fleas over time.

Conclusions: We demonstrate that disparate foregut blockage pathologies exhibited by distinct extant $Y$. pestis strain genotypes do not influence transmission efficiency from $X$. cheopis fleas. In fact, distinct extant $Y$. pestis genotypes maintain equivalently effective blockage and transmission efficiencies which is likely advantageous to maintaining continued successful plague spread and establishment of new plague foci.

Keywords: Yersinia pestis, Biovar, Xenopsylla cheopis, Glycerol, Transmission efficiency

*Correspondence: vvadyvaloo@wsu.edu

Paul G Allen School for Global Animal Health, Washington State

University, Washington 99164, USA

(c) The Author(s) 2020. This article is licensed under a Creative Commons Attribution 4.0 International License, which permits use, sharing, adaptation, distribution and reproduction in any medium or format, as long as you give appropriate credit to the original author(s) and the source, provide a link to the Creative Commons licence, and indicate if changes were made. The images or other third party material in this article are included in the article's Creative Commons licence, unless indicated otherwise in a credit line to the material. If material is not included in the article's Creative Commons licence and your intended use is not permitted by statutory regulation or exceeds the permitted use, you will need to obtain permission directly from the copyright holder. To view a copy of this licence, visit http://creativeco mmons.org/licenses/by/4.0/. The Creative Commons Public Domain Dedication waiver (http://creativecommons.org/publicdomain/ zero/1.0/) applies to the data made available in this article, unless otherwise stated in a credit line to the data. 


\section{Background}

Yersinia pestis, the etiologic agent of bubonic plague, can be transmitted by a flea bite. In this model, the flea siphons blood into the midgut using muscles in its head that act as a peristaltic pump during feeding. The blood is drawn through the foregut which comprises the esophagus and proventriculus (PV), a valvular organ that ensures forward blood passage into the midgut for digestion while simultaneously preventing backflow [1]. However, when the flea acquires an infectious $Y$. pestis blood meal, the bacteria forms a biofilm in the PV that partially or completely obstructs function of this organ and blood passage to the midgut. This is referred to as biofilm-mediated blockage of the flea foregut [2]. During blockage, active ingestion of a subsequent blood meal causes distention of the esophagus and a localized buildup of hydrodynamic pressure in the proximal region of the esophagus immediately preceding the blocked PV [3]. An elastic recoil of the blood-filled esophagus follows, resulting in regurgitation of some $Y$. pestis biofilm back into the bite site thereby facilitating transmission. High blockage rates are thus synonymous with effective transmission, and both partial and complete blockage can cause blockage-mediated regurgitative transmission of $Y$. pestis $[2,4]$.

The biofilm-mediated blockage localized to the PV is comprised of dense multicellular aggregates of $Y$. pestis that are characterized by a self-produced extracellular polymeric substance (EPS). The EPS is primarily composed of poly-N-acetyl-D-glucosamine (PNAG) units [5]. The biosynthetic pathway of PNAG is encoded by the hmsHFRS locus which is positively post-transcriptionally regulated by the second messenger molecule, c-di-GMP. The two functional diguanylate cyclase (DGC) enzymes HmsD and HmsT synthesize c-diGMP. HmsD predominantly affects biofilm production during flea colonization, whereas HmsT plays a lesser role in biofilm formation in the flea environment [6-8].

Historically, Y. pestis has been categorized into three clinically relevant biovars (bvs) differentiated by the biochemical abilities to ferment glycerol and arabinose, and reduce nitrate [9]: Antiqua (glycerol positive, arabinose positive and nitrate positive); Medievalis (glycerol positive, arabinose positive and nitrate negative); and Orientalis (glycerol negative, arabinose positive and nitrate positive). Recent phylogenetic analyses provide enhanced resolution of $Y$. pestis strain characteristics, evolutionary history of biovars and associated lineages, strain geographical pattern distinctiveness, and plague spread $[10,11]$. Additionally, mathematical modelling reveals that branch 1 Orientalis and branch 2 Medievalis lineage strains spread significantly faster than others [12-15].
In laboratory based experimental studies, the prototype model of the interaction between $Y$. pestis strain KIM6+ and Xenopsylla cheopis has forged an understanding of the details regarding $Y$. pestis gut colonization, blockage development, transmission, and the bacterial genetic determinants for this process, including roles of $h m s H$ FRS, hmsT, hmsD and $h m s P$ genes $[6,7,16]$. The KIM6+ strain is a Medievalis bv branch 2 strain that was isolated from a Kurdish Iranian man $[17,18]$. The oriental rat flea, $X$. cheopis, is implicated in past plague pandemics and current plague outbreaks in Madagascar [19] and is reputed to be an efficient vector of $Y$. pestis [20-22].

There remains minimal information regarding how different $Y$. pestis strains influence vector transmission efficiency. Genetic differences in pathogen strains and pathogen fitness are demonstrated determinants for vector transmission efficiency in animal and plant diseases as exemplified in the interactions between Zika virus and Aedes aegypti, bluetongue virus and Culicoides sonorensis midges, and Xylella fastidiosa and sharpshooter leafhoppers [23-25].

Similarly, to understand if strain-specific $Y$. pestis characteristics influence flea transmission efficiency, we comparatively characterized flea infection dynamics using two different $Y$. pestis strains in $X$. cheopis fleas, KIM6+ for which flea infection dynamics are well characterized and CO92. Use of the CO92 strain was motivated by several factors. First, $\mathrm{CO} 92$ belongs to the Orientalis branch 1 lineage comprising globally circulating strains, some directly implicated in causing the highest contemporary plague global case burden [12, 26, 27]. In contrast, Antiqua and Medievalis bvs are typically associated with enzootic rodent species in long term plague foci in Africa and Asia [28]. Secondly, CO92 was originally isolated from a fatal human case of pneumonic plague [29] and is primarily used for experimental infections in a murine plague model [30-32]. Thirdly, the most discerning genetic difference between $\mathrm{CO} 92$ and $\mathrm{KIM} 6+$ is that CO92 is incapable of metabolizing glycerol, allowing testing of a distinguishing metabolic trait in vector competency. Finally, CO92 produces significantly more biofilm than KIM6+ in vitro suggesting that it may have higher blockage rates in fleas [6]. Given these factors, we posited that CO92 may have enhanced abilities to colonize fleas and be transmitted from these insects.

\section{Methods}

\section{Bacterial culture conditions}

Yersinia pestis strains were cultured in heart infusion broth (HIB; BD Difco, New Jersey, USA) at $26{ }^{\circ} \mathrm{C}$ with aeration or grown on heart infusion agar (HIA). When appropriate, media were supplemented with carbenicillin $(100 \mu \mathrm{g} / \mathrm{ml})$, kanamycin $(50 \mu \mathrm{g} / \mathrm{ml})$ or trimethoprim 
(25 $\mu \mathrm{g} / \mathrm{ml})$. All strains, plasmids and oligonucleotides are listed in Tables 1 and 2.

\section{GFP insertion into $Y$. pestis}

Yersinia pestis strains were chromosomally tagged at the $\operatorname{att} \operatorname{Tn} 7$ site with gfpmut3 using a Tn7-based system [33]. To do this, gfpmut3 under the control of the $\mathrm{P}_{\text {trc }}$ promoter was restricted from pGP-Tn7- $\mathrm{P}_{\text {trc }}$ gfpmut3 [34] and cloned into matching restriction sites of pUC18R6KT-mini-Tn7T-Km [33] to create pUC18R6KT-mini-Tn7T-P trc gfpmut3 and electroporated into electrocompetent strains of $Y$. pestis. These plasmid constructs were verified by sequencing. Alternatively, $Y$. pestis electrocompetent strains were transformed with pAcGFP1 and selected on HIA containing carbenicillin.

\section{Deletion of $h m s D$ and $h m s T$}

Deletion of $h m s D$ and $h m s T$ was achieved by homologous recombination using the lambda red recombinase system [35]. Flanking regions of approximately $500 \mathrm{bp}$ upstream and downstream of the $h m s D$ and $h m s T$ genes were first amplified by PCR. The resulting PCR products were gel-purified and combined using splice-overlap extension (SOE) PCR with a kanamycin resistance cassette flanked by frt sites that were previously amplified from the plasmid pKD13 [35]. Electrocompetent $Y$. pestis CO92 carrying $\mathrm{pKOBEG}$ and expressing the recombinase was transformed with purified SOE PCR fragments [36]. Recombinant strains were picked on kanamycin and confirmed by PCR. The kanamycin resistance cassette introduced in the previous step was resolved by the introduction of pFLP3. Deletion mutants CO92 $\Delta h m s D:: f r t$ and CO92 $\Delta h m s T:$ :frt were confirmed by sequencing.

\section{Flea infections}

Yersinia pestis strains confirmed as Pgm-positive were cultured overnight in heart infusion broth (HIB) as described previously [37]. Bacteria were added to $6 \mathrm{ml}$ of sodium heparinized mouse blood (BioIVT, New York, USA) at a concentration of $\sim 1 \times 10^{9} \mathrm{CFU} / \mathrm{ml}$ [38]. Xenopsylla cheopis fleas were then allowed to feed on the infected blood using a previously described artificial feeding apparatus [16, 39]. Only fleas that took full blood meals were selected. Fifty male and 50 female fleas were maintained at $21{ }^{\circ} \mathrm{C}$ and $75 \%$ relative humidity, fed twice weekly on uninfected mice and monitored for proventricular blockage over a period of 28 days as previously described [16]. To perform co-infections, the protocol was followed as previously described [39].

\section{Determining Y. pestis CFU in the infectious blood meal and infected flea samples}

After artificial infection, a sample of the infected blood meal was serially diluted and plated on Columbia blood agar (Hardy Diagnostics, California, USA) to determine $Y$. pestis $\mathrm{CFU} / \mathrm{ml}$ in the blood. To quantify infection rate and bacterial load of the fleas, 20 infected fleas were collected immediately after the infectious blood meal, and at days 7, 14 and 28 post-infection. Fleas were subjected to individual plating on brain heart infusion (BHI) agar (BD Difco) supplemented with $1 \mu \mathrm{g} / \mathrm{ml}$ irgasan and $10 \mu \mathrm{g} / \mathrm{ml}$ hemin to determine CFU count. To determine CFU/flea from co-infected fleas, samples were treated as previously described, but plated on heart infusion agar with and without $50 \mu \mathrm{g} / \mathrm{ml}$ kanamycin to select for KIM6+::kan ${ }^{R}$ on individual sets of plates respectively [39].

\section{Imaging and esophageal scoring of fleas}

Blocked fleas were dissected in PBS to isolate the flea gut. A $1.5 \mathrm{~mm}$ glass cover slip was then placed over the samples for imaging. Flea guts were imaged with a Leica DMi8 epifluorescence microscope (Leica Microsystems, Illinois, USA) with phase contrast and a GFP filter cube (Ex. 470/40nm, Em. 525/50nm). Esophageal images were first evaluated for valid scoring by determining if: (i) the esophagus was intact; and (ii) the entire esophagus was visible. If these criteria were met, the esophagus was scored for presence of GFP in which presence of GFP in $<1 / 3$ the length of the esophagus was assigned a proximal colonization status, and presence of GFP in $\geq 1 / 3$ the length of the esophagus was assigned a distal-medial colonization status.

\section{Mass transmission experiment}

For transmission experiments, fleas that took an infectious blood meal were housed as described above. On days 7 and 14, the fleas involved in the transmission experiment fed on $5 \mathrm{ml}$ of sterile sodium heparinized CD-1 (ICR) mouse blood (BioIVT) using the artificial feeding system. After $60 \mathrm{~min}$, fleas were examined microscopically to determine how many had taken a blood meal, and of those, how many were blocked. Transmission experiments were performed as previously described [40]. Immediately after each transmission feeding, all 5 $\mathrm{ml}$ of blood were removed and plated on Columbia blood agar plates. The interior of the feeder was rinsed 24 times with $1 \mathrm{ml}$ of PBS and these washes were pooled. Pooled washes were centrifuged at $\sim 12,000 \times g$ for $10 \mathrm{~min}$, supernatant removed, and the pellet was plated onto Columbia blood agar plates. The external surface of the mouse skin was disinfected with $70 \%$ ethanol twice to reduce surface contaminants and cut into small pieces that were homogenized in PBS in a FastPrep homogenizer (MP 
Table 1 List of strains and plasmids used

\begin{tabular}{|c|c|c|}
\hline Yersinia pestis strains and plasmids used & Characteristics & Reference \\
\hline $\operatorname{cog} 2$ & Pgm + pCD1- pMT1+ pPCP1+, parental strain & [32] \\
\hline CO92 glmS-pstS::P $P_{t r c} G F P$ & CO92 with chromosomally inserted gfp for imaging & This study \\
\hline CO92 glpD+ & Defective $g / p D^{\prime}$ allele replaced with functional $g / p D$ gene from KIM6+ in the chromosome & [32] \\
\hline CO92 glpD+ glmS-pstS::P trc GFP & $\begin{array}{l}\text { defective } g / p D^{\prime} \text { allele replaced with functional glpD gene in the chromosome, with chro- } \\
\text { mosomally inserted GFP for imaging }\end{array}$ & This study \\
\hline Co92gly & $\begin{array}{l}\text { Defective copy of the } g / p D^{\prime} \text { allele replaced with functional } g / p D \text { in the chromosome, and } \\
\text { carries glpFKX from KIM6+ on pTpGLPFKX6 }\end{array}$ & This study \\
\hline CO92gly gImS-pstS::P trc GFP & CO92gly with chromosomally inserted gfp for imaging & This study \\
\hline CO92 $\triangle h m s D:: f r t$ & $\triangle h m s D$ & This study \\
\hline CO92 $\triangle h m s T:: f r t$ & $\Delta h m s T$ & This study \\
\hline KIM6+ & Pgm+ pCD1-pMT1+ pPCP1+, parental strain & [16] \\
\hline $\mathrm{KIM} 6+:: \mathrm{kan}^{R}$ & KIM6+ with chromosomally inserted kanamycin resistance for co-infections & [39] \\
\hline KIM6+ pAcGFP1 & KIM6+ with GFP for imaging & This study \\
\hline pAcGFP1 & Carries AcGFP1, Cbr & $\begin{array}{l}\text { Clontech (Moun- } \\
\text { tain View, CA, } \\
\text { USA) }\end{array}$ \\
\hline pTNS2 & TnsABC+D specific transposition pathway, $\mathrm{Cb}^{r}$ & [33] \\
\hline pKD13 & Source of kanamycin resistant cassette and flanking Frt sequence & [35] \\
\hline pFLP3 & Recombinase, $\mathrm{Cb}^{r}$, Tet ${ }^{r}$ & [33] \\
\hline pUC18R6KT-mini-Tn7T-Km & Cloning vector for $\mathrm{Tn} 7$ insertion, $\mathrm{Cb}^{r}, \mathrm{Km}^{\mathrm{r}}$ & [33] \\
\hline pGP-Tn7-P tre 9 fpmut3 & Source of $\mathrm{P}_{\mathrm{trc}} g$ fpmut $3, \mathrm{Cb}^{\mathrm{r}}$ & [34] \\
\hline pUC18R6KT-mini-Tn7T-P trc $g$ fpmut3 & Cloning vector for $\mathrm{Tn} 7$ insertion of $\mathrm{P}_{\mathrm{trc}}$ gfpmut3, $\mathrm{Cb}^{\mathrm{r}}, \mathrm{Km}^{\mathrm{r}}$ & This study \\
\hline
\end{tabular}

Abbreviations: $\mathrm{Cb}^{\mathrm{r}}$, carbenicillin resistance; $\mathrm{Km}^{\mathrm{r}}$, kanamycin resistance; Tet', tetracycline

Table 2 List of oligonucleotides used

\begin{tabular}{llll}
\hline Oligonucleotide & Reference & Purpose & Sequence \\
\hline p130 & {$[35]$} & Amplify kanamycin cassette and check insertion & GTGTAGGCTGGAGCTGCTTC \\
p131 & {$[35]$} & Amplify kanamycin cassette and check insertion & ATTCCGGGGATCCGTCGACC \\
p407/pGPTn7pro & This study & To clone gfpmut3 into pUC18R6KT-mini-Tn7T-Km & ATAGGAATTCCTTCTCGAGCGATGCACGGTGC \\
p408/pGFPTn7R & This study & To clone gfpmut3 into pUC18R6KT-mini-Tn7T-Km & CAGAGCGCTTTTGAAGCTAATTCGATC \\
p544 & {$[51]$} & To mutate $h m s T$ & ACGTGGTACAACATGCTGACGGTTG \\
p545 & {$[51]$} & To mutate $h m s T$ & GAAGCAGCTCCAGCCTACACCATAATATCGTGCTGTCAGTAGACTAAT \\
& & & AAATC \\
p546 & {$[51]$} & To mutate $h m s T$ & GGTCGACGGATCCCCGGATGATTAACTCACTGAACATACGGACGCTC \\
& & TATG \\
p547 & {$[51]$} & To mutate $h m s T$ & CTTCTCATCATCATCTGATGCGGCC \\
p548 & {$[51]$} & To mutate $h m s D$ & GCCAGTAATATCAATGCAGATATTCTGCG \\
p549 & {$[51]$} & To mutate $h m s D$ & GAAGCAGCTCCAGCCTACACCATAGATTGGTTTTTATCGTGTTCATCGT \\
p550 & {$[51]$} & To mutate $h m s D$ & GGTCGACGGATCCCCGGATGTGACATGCAGAAAATGCAAAACAA \\
p551 & {$[51]$} & To mutate $h m s D$ & GCGTT \\
p556 & This study & To verify $h m s D$ mutation & CGTTAGGACGCGGTGATAATAATGGCG \\
p577 & This study & To verify $h m s D$ mutation & AGCGCAGAATTCGTACCTAACGCCGATTCAACC \\
p578 & This study & To verify $h m s T$ mutation & ATCCACTTCATCCTGGCAGC \\
p579 & This study & To verify $h m s T$ mutation & CGCAAGACTCTCGCTTCTTTG \\
\hline
\end{tabular}


Biomedicals, California, USA) to release any bacteria deposited by flea bite into the mouse skin. Skin sample supernatants were pooled and treated in the same manner as the pooled washes. After incubation at $28^{\circ} \mathrm{C}$ for 48 $\mathrm{h}, Y$. pestis colonies were counted and confirmed by replicate patch plating on both Yersinia selective agar plates and Congo Red agar. At each time point post-infection 20 fleas were collected for enumeration of bacterial loads. To maintain the normal bi-weekly schedule of feeding, fleas were also fed on neonatal mice on days 4 and 11 .

\section{Statistical analysis}

All analyses were performed using GraphPad Prism 7 (GraphPad Software Inc., La Jolla, California, USA.). Fisher's exact test was performed with the fisher.multcomp package in $\mathrm{R}$ (3.6.1). The statistical tests that were used and relevant $P$-values are indicated in the figure legends and results.

\section{Results \\ KIM6+ and CO92 infected X. cheopis fleas have similar blockage rates}

To determine if $Y$. pestis strain specific distinctions can be made in the interactions of $Y$. pestis and its prototype flea vector, $X$. cheopis, blockage rates and flea colonization dynamics were directly compared between CO92 and KIM6+ strains. The distinctive phenotypic difference between KIM6+ and CO92 is the inability of CO92 to metabolize glycerol. Therefore, to simultaneously determine the role of glycerol utilization in potential differences that may be uncovered in flea infection dynamics between $\mathrm{CO} 92$ and $\mathrm{KIM} 6+$, we also tested a previously reported CO92 strain [32] that was restored in ability to metabolize glycerol (referred to as CO92gly). For this, $X$. cheopis fleas were artificially infected with CO92, KIM6+ or CO92gly strains to determine blockage rate, flea infection rate, and bacterial burden per flea over time. We found that the cumulative blockage rate of fleas infected with KIM6+ or CO92 over the period of a month averaged approximately 37\% (SD $\pm 3.00 \%$ ), and $40 \%$ (SD \pm $5.78 \%$ ) respectively (Fig. 1a). This was consistent with previous reports of blockage rates between $25-45 \%$ for the KIM6+ strain [40]. Strikingly, mean blockage rate of CO92gly infected fleas was $54 \%$ (SD $\pm 7.5 \%$ ), significantly higher (t-test: $t_{(4)}=4.143, P=0.0143$ ) than blockage rates for KIM6+ (Fig. 1a). However, no significant differences in bacterial burden per flea and rate of flea infection were noted for all three strains (Fig. 1b).

A detailed assessment of temporal incidence of blockage demonstrated that peak incidence of blockage occurred at 14.3 days post-infection for KIM6+ (Fig. 1c), but at 11 days post-infection for CO92 (Fig. 1d). In contrast, temporal incidence of blockage did not follow a normal distribution and was predicted to peak at a physiologically unrealistic 0.15 days ( $\sim 3 \mathrm{~h}$ post-infection) for CO92gly (Fig. 1e).

\section{KIM6+ biofilm blockage extends the full length of the $X$. cheopis esophagus}

No quantitative differences in flea blockage rate, mortality, or infection were noted between the KIM6+ and CO92 strains. However, in preliminary studies we noted that KIM6+ colonized the entire length of the flea esophagus, as well as the PV, whereas CO92 primarily colonized the PV alone. Microscopic visualization prior to application of a coverslip eliminated any concerns that the phenotype observed resulted from the process of the coverslip laying down on the gut. To quantitatively assess this phenotype, we developed an esophageal pathology scoring rubric which enabled parsing of colonization status into major (distal-medial) and minor (proximal) with respect to $Y$. pestis esophagus colonization of the flea foregut. Intriguingly and consistent with preliminary observations, a significantly greater (Fisher's exact test: OR: $0.2534,95 \%$ CI: $0.1164-0.5457, P<0.0001) 65 \%$ of KIM6+ blocked fleas compared to only $32 \%$ of fleas blocked with CO92 colonized most of the surface of the flea esophagus (Fig. 2a-e). The CO92gly strain maintained a similar foregut colonization phenotype to the parental strain (Fisher's exact test: OR: 1.339, 95\% CI: $0.6113-3.069, P=0.4360)$.

\section{Transmission of KIM6+, CO92 and CO92gly is not significantly different}

Full esophagus colonization by the sticky $Y$. pestis biofilm reflects that bacteria are in closer proximity to the feeding mouthparts and can likely be more efficiently regurgitated back into the flea bite site. Alternatively, the impediment caused by full $Y$. pestis esophageal colonization may reduce build-up of the hydrodynamic forces and pressure in the esophagus, resulting in fewer $Y$. pestis cells being dislodged and regurgitated from the 
a

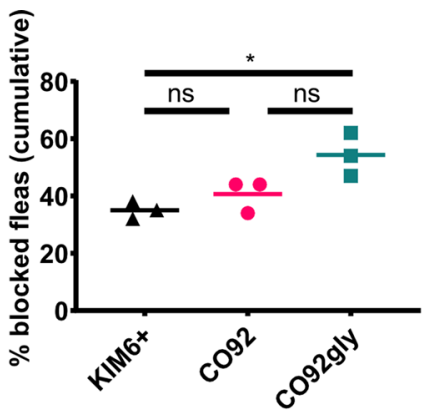

b

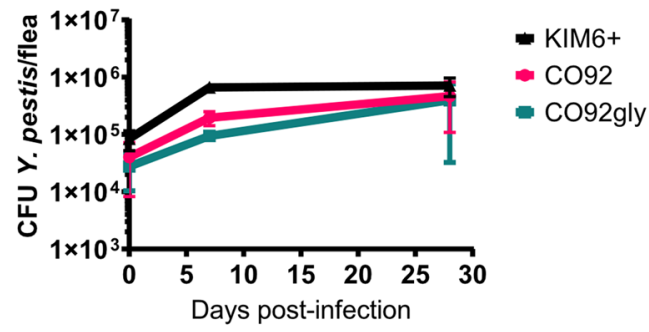

C

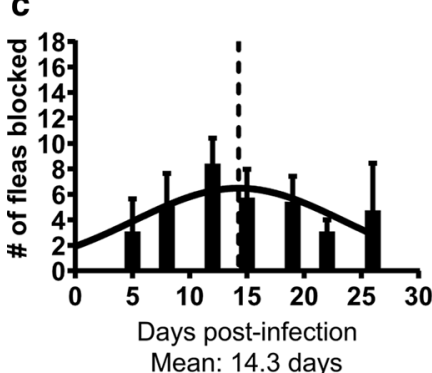

d

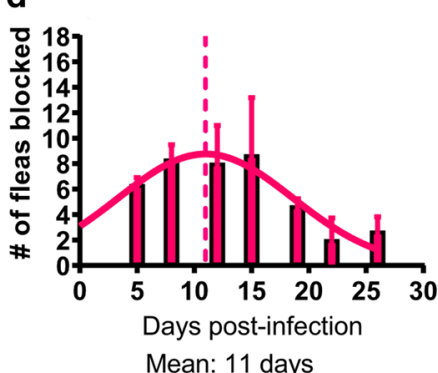

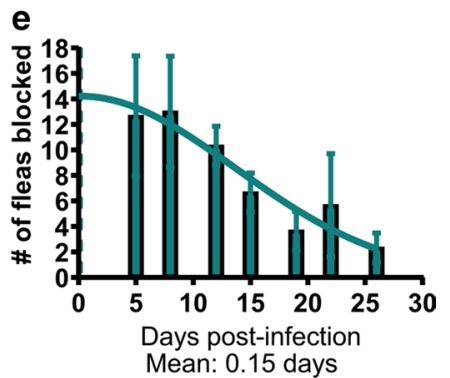

Fig. 1 a Foregut blockage and infection dynamics in fleas infected with CO92, KIM6+ and CO92gly. Cumulative flea blockage rate of strains KIM6+ (black), CO92 (pink) and CO92gly (teal). Error bars represent the mean of three independent flea infection experiments per strain. ${ }^{*} P \leq 0.05 . \mathbf{b}$ Growth kinetics of strains KIM6+, CO92, and CO92gly during infection of X. cheopis fleas, error bars represent the mean \pm SD of three independent flea infections per strain with each time point reflecting 15-20 fleas per strain per independent experiment. Histograms representing temporal incidence of blockage in KIM6+ (c), CO92 (d), and CO92gly (e) were fit to a Gaussian model, and the dashed line indicates mean time to peak blockage. CO92gly had an earlier mean to peak blockage than KIM6+ or CO92

biofilm blockage. To address this, we asked if the esophageal colonization differences observed among the CO92, CO92gly and KIM6+ strains might impact regurgitative transmission of bacteria into the flea bite site. Cohorts of $X$. cheopis fleas were infected with the $Y$. pestis strains of interest. Once a week for two weeks, mass-transmission experiments were conducted to determine the total CFU of each $Y$. pestis strain that is regurgitated by infected fleas into an artificial blood-feeding apparatus containing sterile blood. For all the strains tested, KIM6+, CO92 and CO92gly, a wide and variable range of $Y$. pestis CFU was transmitted per feeding (Fig. 3, Table 3). Although not statistically significant, fewer CO92gly CFUs were transmitted per blocked flea bite with similar numbers of blocked fleas occurring in all three strains (Fig. 3a,
Table 3). Similarly, fewer CO92gly CFUs were transmitted per infected flea bite (Fig. 3b).

\section{KIM6+ and CO92 are equally fit during competitive flea co-infection}

The distinctive flea gut colonization phenotypes exhibited by the CO92 and KIM6+ strains may be important traits for flea infection that can only be discerned in more sensitive competitive fitness studies [37, 39]. To test this, we infected fleas with a 1:1 ratio of both strains. At $2 \mathrm{~h}$ post-infection, and by days 7 and 14 post-infection, there was no significant change in the ratio of each strain per flea. This data implies that KIM6+ and CO92 are equally fit during competitive co-infection in fleas (Fig. 4). 


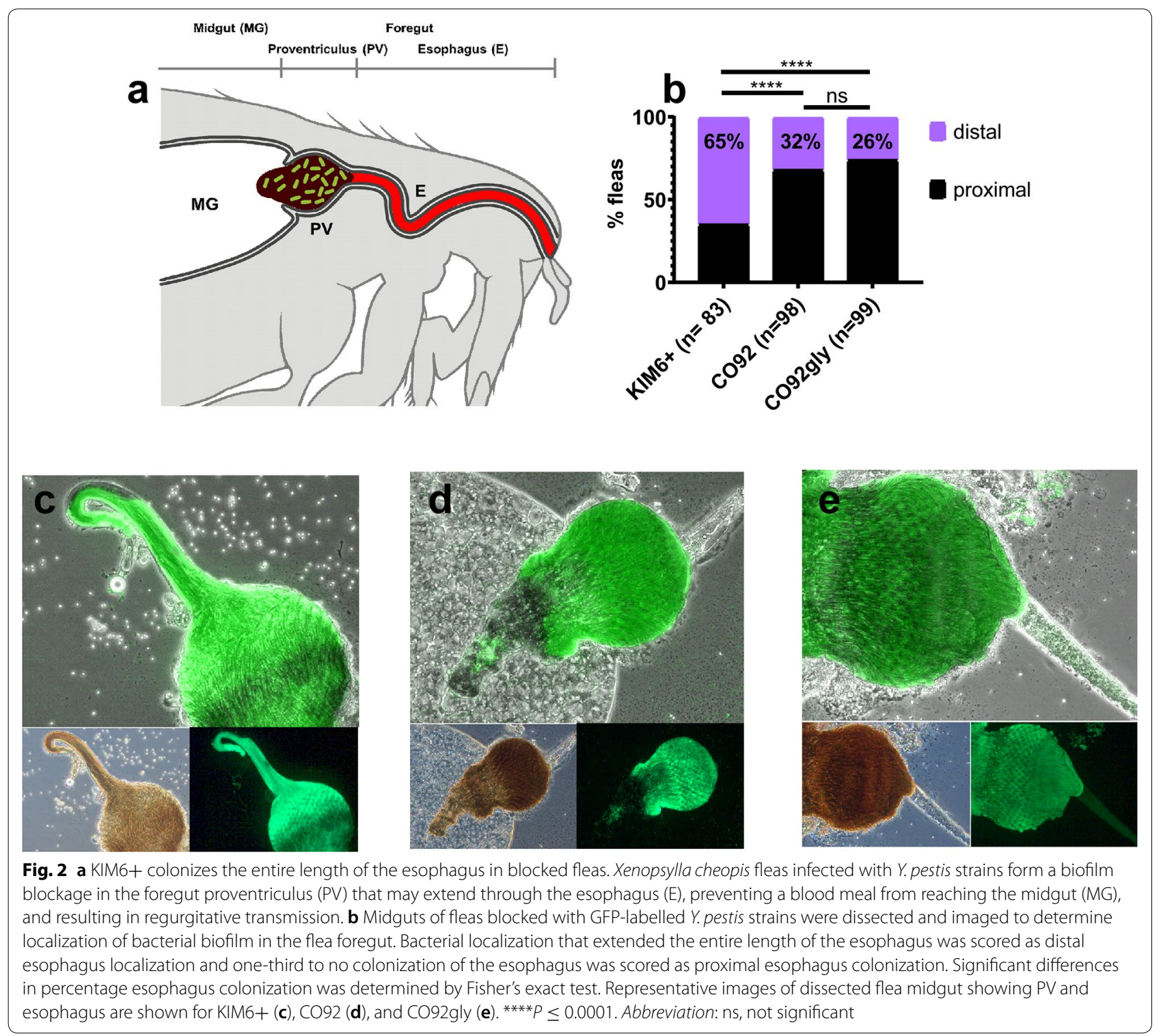

CO92 hmsT and $h m s D$ diguanylate cyclase encoding genes have matching roles in blockage formation to KIM6+ hmsT and $h m s D$ genes

We additionally considered that the phenotypic differences in foregut colonization noted between KIM6+ and CO92 was a consequence of disparate nutritional sensing due to the biochemical differences in the strains. Localized environmental signals including nutrient signals in the flea foregut may contribute to differential regulation of the diguanylate cyclase encoding genes [41-43] and alterations in c-di-GMP signaling and biofilm production. We know that in the KIM6+ strain, the hmsD gene is essential for blockage while the absence of $h m s T$ reduced blockage rates by approximately 50\% [7]. However, the individual contributions of $\mathrm{HmsD}$ and $\mathrm{Hms} \mathrm{T}$ diguanylate cyclases to blockage development in CO92 strains is unknown [7]. We therefore compared blockage rates between CO92 mutants in $h m s T$ and $h m s D$ alongside the isogenic parental CO92 strain and found an $82 \%$ and $39 \%$ reduction in blockage for mutants relative to the 

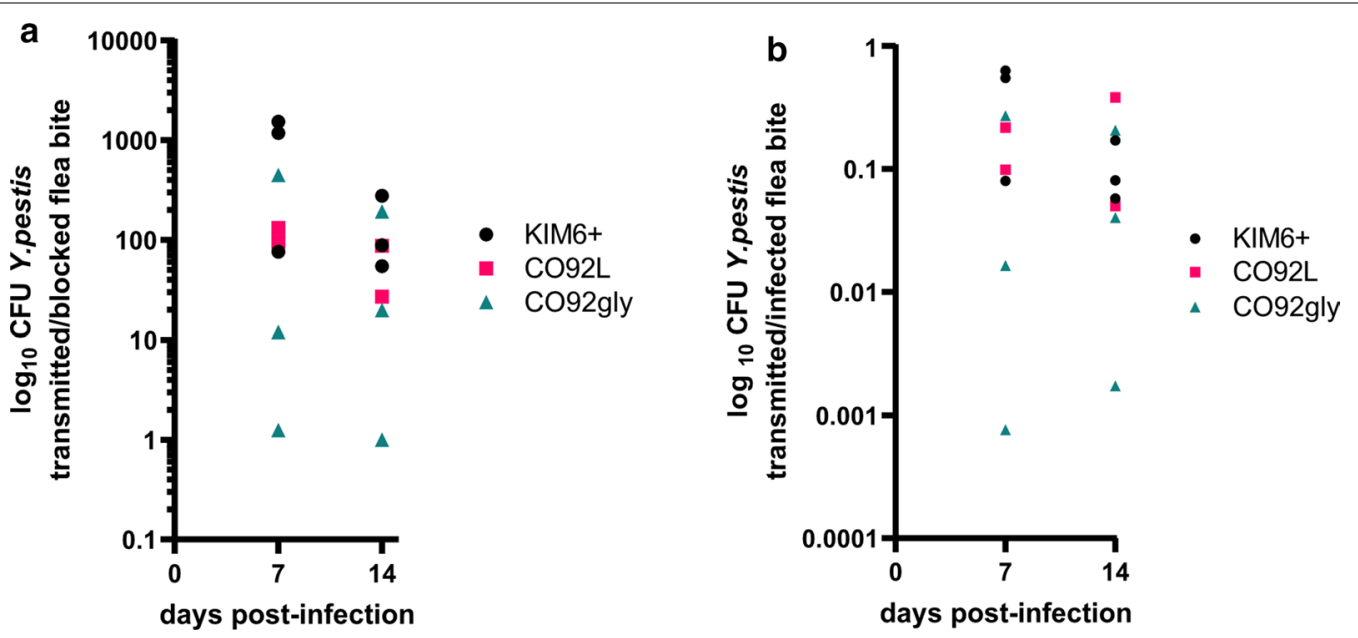

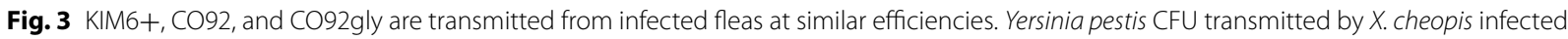
with KIM6+ (black triangles), CO92 (pink circles), and CO92gly (teal squares) during in vitro mass transmission experiments, per blocked flea bite (total number of transmitted CFUs/ number of blocked fleas) (a) and per infected flea bite (total number of transmitted CFUs/[number of fed fleas $\times \%$ infected fleas]) (b). Fleas were fed on mouse blood seeded with $\sim 10^{9}$ Y. pestis CFU/ml and were subjected to mass artificial transmission experiments 7 and 14 days after a single infectious blood meal. Symbols represent three independent experiments for KIM6+ and CO92gly, and two independent experiments for CO92. No significant difference in CFU Y. pestis transmitted per blocked flea or per infected flea bite between strains was noted using one-way ANOVA analysis

CO92 parental strain, respectively (Table 4 ). The roles of $\mathrm{Hms} \mathrm{T}$ and HmsD in blockage formation by CO92, as such, recapitulated that occurring in the KIM6+ strain (Table 4).

\section{Discussion}

The present study aimed to understand the contribution of genetically and biochemically distinct $Y$. pestis strains to vector competence of the prototype flea vector species of plague, $X$. cheopis [37]. Our studies reveal that pathogen-specific differences in colonization of the flea foregut between the CO92 and KIM6+ strains do not result in differences in blockage rates, competitive fitness, in vitro transmission efficiency, or demonstrate different contributions of diguanylate cyclases to blockage during infection of $X$. cheopis fleas. The present blockage rate data of CO92 to KIM are consistent with previous results with two other Orientalis strains, 195/P (16) and GB [44].

In this study we additionally examined whether the ability to utilize glycerol, present in KIM6+ but absent in CO92, may contribute to vector competence. This was motivated by previous work demonstrating that genes encoding glycerol uptake are specifically induced in KIM6+ blocked fleas suggesting that active glycerol metabolism occurs in this strain during flea infection [37,
45]. Loss of glycerol uptake and utilization in CO92 is due to mutations in $g l p D$ and the $g l p F K X$ operon, but a previous study undertook restoration of this biochemical ability by complementing the $\mathrm{CO} 92$ strain with intact copies of $g l p D$ and the $g l p F K X$ genes [32]. Functionally restoring glycerol utilization to the CO92 wild-type strain did not impact virulence in a mouse model or biofilm production in vitro, suggesting that glycerol utilization is not essential for these functions [32].

Our study attributes re-establishment of glycerol utilization in the CO92gly strain to enhanced blockage rates but not changes in growth, flea bacterial burdens, or esophageal colonization in fleas. Principally, blockage is dependent on production of a biofilm EPS matrix that allows a robust adherence of bacterial aggregates to the PV a few days post-acquisition of the infected blood meal [46]. EPS production likely occurs at elevated levels in CO92gly leading to hyperbiofilm production in the flea gut. This may be counterproductive to transmission if the cohesive nature of the hyperbiofilm prevents easy dislodging of bacteria by hydrodynamic forces, resulting in lower numbers of regurgitated bacteria. Interestingly, CO92gly-infected fleas tended to transmit fewer bacteria despite a significantly higher blockage rate. The phosphodiesterase activity of $Y$. pestis HmsP is required 
Table 3 Xenopsylla cheopis mass transmission summary

\begin{tabular}{|c|c|c|c|c|c|}
\hline Days PI & No. of fleas fed & $\%$ infected & CFU/infected flea & No. of fleas blocked & CFU transmitted \\
\hline \multicolumn{6}{|c|}{ X. cheopis infected with KIM6+ mass transmission summary } \\
\hline \multicolumn{6}{|c|}{ Experiment $1\left(1.10 \times 10^{9} \mathrm{CFU} / \mathrm{ml}\right.$ blood $)$} \\
\hline 0 & - & 80 & $1.20 \mathrm{E}+05$ & - & - \\
\hline 7 & 80 & 95 & $6.85 E+05$ & 8 & 607 \\
\hline 14 & 88 & 100 & $7.81 \mathrm{E}+05$ & 13 & 710 \\
\hline \multicolumn{6}{|c|}{ Experiment $2\left(2.46 \times 10^{9} \mathrm{CFU} / \mathrm{ml}\right.$ blood $)$} \\
\hline 0 & - & 100 & $2.19 \mathrm{E}+05$ & - & - \\
\hline 7 & 206 & 95 & $6.35 E+05$ & 11 & 10,749 \\
\hline 14 & 120 & 95 & $4.50 \mathrm{E}+05$ & 7 & 1942 \\
\hline \multicolumn{6}{|c|}{ Experiment $3\left(6.70 \times 10^{8} \mathrm{CFU} / \mathrm{ml}\right.$ blood $)$} \\
\hline 0 & - & 100 & $1.08 \mathrm{E}+05$ & - & - \\
\hline 7 & 206 & 100 & $2.82 \mathrm{E}+05$ & 7 & 12,957 \\
\hline 14 & 120 & 90 & $2.69 \mathrm{E}+05$ & 7 & 620 \\
\hline \multicolumn{6}{|c|}{ X. cheopis infected with CO92 mass transmission summary } \\
\hline \multicolumn{6}{|c|}{ Experiment $1\left(1.03 \times 10^{9} \mathrm{CFU} / \mathrm{ml}\right.$ blood $)$} \\
\hline 0 & - & 100 & $2.76 \mathrm{E}+03$ & - & - \\
\hline 7 & 132 & 90 & $1.11 \mathrm{E}+05$ & 27 & 2583 \\
\hline 14 & 65 & 95 & $3.46 \mathrm{E}+05$ & 27 & 2353 \\
\hline \multicolumn{6}{|c|}{ Experiment $2\left(2.27 \times 10^{9} \mathrm{CFU} / \mathrm{ml}\right.$ blood $)$} \\
\hline 0 & - & 100 & $8.87 \mathrm{E}+04$ & - & - \\
\hline 7 & 183 & 95 & $2.62 \mathrm{E}+05$ & 13 & 1714 \\
\hline 14 & 81 & 100 & $3.37 \mathrm{E}+05$ & 15 & 405 \\
\hline \multicolumn{6}{|c|}{ X. cheopis infected with CO92gly mass transmission summary } \\
\hline \multicolumn{6}{|c|}{ Experiment $1\left(2.06 \times 10^{9} \mathrm{CFU} / \mathrm{ml}\right.$ blood $)$} \\
\hline 0 & - & 95 & $2.51 \mathrm{E}+04$ & - & - \\
\hline 7 & 103 & 85 & $4.04 \mathrm{E}+05$ & 12 & 143 \\
\hline 14 & 52 & 85 & $1.81 \mathrm{E}+05$ & 9 & 177 \\
\hline \multicolumn{6}{|c|}{ Experiment $2\left(1.45 \times 10^{9} \mathrm{CFU} / \mathrm{ml}\right.$ blood $)$} \\
\hline 0 & - & 65 & $1.19 \mathrm{E}+04$ & - & - \\
\hline 7 & 92 & 90 & $7.14 \mathrm{E}+05$ & 5 & 2233 \\
\hline 14 & 54 & 75 & $8.94 \mathrm{E}+04$ & 7 & 7 \\
\hline \multicolumn{6}{|c|}{ Experiment $3\left(6.70 \times 10^{8} \mathrm{CFU} / \mathrm{ml}\right.$ blood $)$} \\
\hline 0 & - & 95 & $7.00 \mathrm{E}+04$ & - & - \\
\hline 7 & 88 & 75 & $7.32 \mathrm{E}+05$ & 4 & 5 \\
\hline 14 & 55 & 85 & $9.45 E+05$ & 5 & 961 \\
\hline
\end{tabular}

Abbreviation: PI, post-infection

to degrade c-di-GMP and decrease EPS production $[6,47]$ and an HmsP mutant of $Y$. pestis is a hyperbiofilm producer generating bacterial aggregates that are extraordinarily cohesive [47]. Alteration of c-di-GMP levels through regulation of its synthesis and degradation enzymes is suggested to occur through sensing of nutritional signals [37, 43], which, in this case could be conferred by the ability to ferment glycerol that is normally absent in $\mathrm{CO} 92$ strains. Glycerol utilization in the $\mathrm{CO} 92$ genetic background does not, however, appear to bode well for optimal transmission efficiency. First, the peak blockage incidence of the CO92gly strain three hours post-infection is physiologically improbable for EPS synthesis. Secondly, this improbable peak blockage incidence and blockage rate strays from the norm exhibited by the extant, circulating epidemic strains, CO92 and 


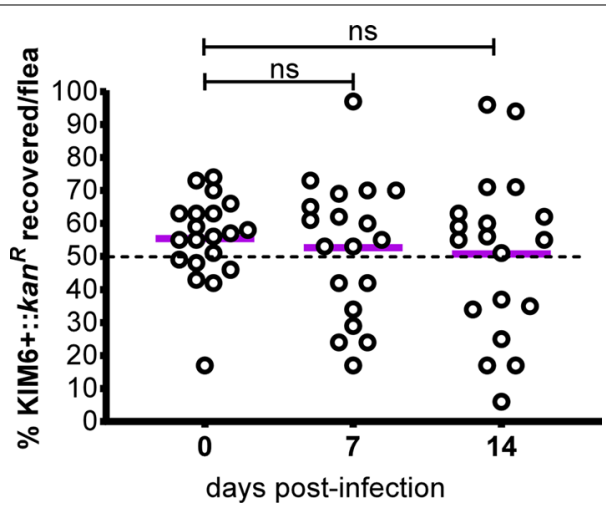

Fig. 4 KIM6+ and CO92 are equally fit during competitive co-infection in fleas. Xenopsylla cheopis fleas were co-infected with a 1:1 ratio of CO92 and KIM6+:"kan ${ }^{R}$. The Y. pestis bacterial loads were determined for 18-20 fleas at time points 0 ( $2 \mathrm{~h}$ post-acquisition of the co-infected blood meal), 7 and 14 days post-infection. The percentage of $\mathrm{KIM} 6+: . \mathrm{kan}^{R}$ in the co-infection was determined and plotted for each time point. One of two independent experiments with similar outcomes is shown. A Student's t-test was used to determine statistical significance between time points. Abbreviation: ns, not significant

KIM6+. Thirdly, this strain shows a tendency to transmit fewer bacteria reflecting reduced transmission efficiency. Marked genome rearrangements also distinguish CO92 from KIM strains and may contribute to gene expression alterations relevant to metabolic processes in these strains [17]. Incorporation of an ability to metabolize glycerol within the existing $\mathrm{CO} 92$ metabolic networks may thus be deleterious to a metabolic physiology that supports optimal flea transmission competency. In KIM6+ glycerol utilization is likely metabolically innocuous. This may explain the loss of the pathway in Orientalis strains which have achieved global distribution.

One significant observation in this study is the predominant occurrence of extended colonization of the esophagus in KIM6+ blocked fleas. The physical anatomy of the flea foregut with regards to musculature surrounding the PV and the width of both the PV and esophagus have proven to be important determinants of flea vector transmission efficiency $[40,48]$. The observation that the KIM6+ biofilm blockage 'infiltrates' the esophagus was made previously and described to be responsible for distending the esophagi width of $X$. cheopis and Oropsylla montana fleas [40]. The wider O. montana esophagus facilitates regurgitative transmission of greater numbers of bacteria in keeping with its greater width in these reports. However, our results did not reveal any difference in the CFU transmitted by KIM6+ and CO92 blocked $X$. cheopis fleas. In fact, this finding is consistent with previous studies that show similar variable ranges of bacteria being transmitted from $X$. cheopis fleas infected with KIM6+, and that transmission by flea bite is a relatively inefficient process requiring bolstering by high flea indices [38, 49]. We speculate that the relevance of esophageal colonization differences regarding regurgitative transmission may differ in other flea species with anatomical foregut characteristics that are distinct from that of $X$. cheopis. Certainly, the proposition that natural endemic foci harbor discrete co-evolved $Y$. pestis strainflea vector species associations is a provocative one that may give credence to this notion $[10,50]$.

\section{Conclusions}

Collectively, our study has highlighted that pathogenspecific traits occur during flea colonization, yet these differences cumulatively amount to comparable efficiencies in X. cheopis fleas to transmit both extant CO92 and KIM6+ strains. This is consistent with the idea that it is evolutionarily favorable for this pathogen to non-selectively be transmitted from a broad range of competent flea vectors. In the backdrop of highly complex plague ecology, further analyses are needed to have a broad understanding of the nuances in vector competence that supports natural maintenance of this pathogen in flearodent cycles worldwide.

Table 4 CO92 c-di-GMP diguanylate cyclases have similar contributing roles to blockage formation as their homologs in KIM6+

\begin{tabular}{|c|c|c|c|c|c|c|}
\hline & \multicolumn{6}{|c|}{ Y. pestis strain } \\
\hline & CO92 & CO92 $\triangle h m s D:: f r t$ & CO92 $\triangle h m s T:: f r t$ & $\mathrm{KIM6}^{\mathrm{a}}$ & $\mathrm{KIM} 6+\Delta h m s D^{\mathrm{a}}$ & $\mathrm{KIM} 6+\Delta h m s T^{\mathrm{a}}$ \\
\hline $\begin{array}{l}\text { Mean } \% \text { reduction in mutant } \\
\text { blockage vs parental strain }\end{array}$ & na & 82 & 39 & na & 92 & 57 \\
\hline Mean CFU/flea (0 dpi) & $3.1 \times 10^{4}$ & $9.4 \times 10^{3}$ & $2.3 \times 10^{4}$ & $1.3 \times 10^{5}$ & $5.1 \times 10^{4}$ & $5.2 \times 10^{4}$ \\
\hline Mean CFU/flea (28 dpi) & $1.3 \times 10^{6}$ & $7.9 \times 10^{5}$ & $3.7 \times 10^{5}$ & $5.4 \times 10^{5}$ & $3.0 \times 10^{5}$ & $4.7 \times 10^{5}$ \\
\hline
\end{tabular}

a All KIM6+ data derived from [7]

Notes: Data is reflective of one experiment, 20 fleas plated per time point

Abbreviations: na, not applicable; dpi, days post-infection 


\section{Abbreviations}

PV: proventriculus; EPS: extracellular polymeric substance; SD: standard deviation; PNAG: poly-N-acetyl-D-glucosamine; DGC: diguanylate cyclase; bvs: biovars; HIB: heart infusion broth; HIA: heart infusion agar; SOE: splice-overlap extension; CFU: colony forming units; GFP: green fluorescent protein; Ex: excitation; Em: emission.

\section{Acknowledgements}

We thank Vladimir Motin for the CO92 glpD+ strain and pTpGLPFKX6 plasmid.

\section{Authors' contributions}

$\mathrm{W}$ and $\mathrm{AL}$ wrote the manuscript. $\mathrm{AL}$, JS and $\mathrm{W}$ analyzed and interpreted data. $\mathrm{AL}$, JS and KG conducted experiments. All authors read and approved the final manuscript.

\section{Funding}

This study was funded by Washington State University CVM Intramural Funds and the National Institutes of Health grant \# R01Al117016-01A1.

\section{Availability of data and materials}

All data generated or analyzed during this study are included in this published article.

\section{Ethics approval and consent to participate}

Use of mice in this study was performed in strict accordance with the recommendations by the National Research Council's Guide for the Care and Use of Laboratory Animals (8th edition) and approved by the Washington State University Institutional Animal Care and Use Committee.

\section{Consent for publication}

Not applicable.

\section{Competing interests}

The authors declare that they have no competing interests.

Received: 7 March 2020 Accepted: 24 June 2020

Published online: 01 July 2020

\section{References}

1. Snodgrass RE. The feeding apparatus of biting and sucking insects affecting man and animals. Washington: Smithsonian Miscellaneous Collections; 1944. p. 1-107.

2. Bacot AW, Martin CJLX. Observations on the mechanism of the transmission of plague by fleas. J Hyg. 1914;13(Suppl.):423-39.

3. Hinnebusch BJ, Jarrett CO, Bland DM. "Fleaing" the plague: adaptations of Yersinia pestis to its insect vector that lead to transmission. Annu Rev Microbiol. 2017;71:215-32.

4. Bacot AW. Further notes on the mechanism of the transmission of plague by fleas. J Hyg. 1915;14(Suppl.): 774-6.3.

5. Bobrov AG, Kirillina O, Forman S, Mack D, Perry RD. Insights into Yersinia pestis biofilm development: topology and co-interaction of $\mathrm{Hms}$ inner membrane proteins involved in exopolysaccharide production. Environ Microbiol. 2008;10:1419-32.

6. Bobrov AG, Kirillina O, Vadyvaloo V, Koestler BJ, Hinz AK, Mack D, et al. The Yersinia pestis HmsCDE regulatory system is essential for blockage of the oriental rat flea (Xenopsylla cheopis), a classic plague vector. Environ Microbiol. 2015;17:947-59.

7. Sun YC, Koumoutsi A, Jarrett C, Lawrence K, Gherardini FC, Darby C, et al. Differential control of Yersinia pestis biofilm formation in vitro and in the flea vector by two c-di-GMP diguanylate cyclases. PLOS ONE. 2011;6:e19267.

8. Bobrov AG, Kirillina O, Ryjenkov DA, Waters CM, Price PA, Fetherston JD, et al. Systematic analysis of cyclic di-GMP signalling enzymes and their role in biofilm formation and virulence in Yersinia pestis. Mol Microbiol. 2011;79:533-51

9. Zhou DS, Tong ZZ, Song YJ, Han YP, Pei DC, Pang X, et al. Genetics of metabolic variations between Yersinia pestis biovars and the proposal of a new biovar, microtus. J Bacteriol. 2004;186:5147-52.
10. Hinnebusch BJ, Chouikha I, Sun YC. Ecological opportunity, evolution, and the emergence of flea-borne plague. Infect Immun. 2016:84:1932-40.

11. Morelli G, Song Y, Mazzoni CJ, Eppinger M, Roumagnac P, Wagner DM, et al. Yersinia pestis genome sequencing identifies patterns of global phylogenetic diversity. Nat Genet. 2010;42:1140-3.

12. $X u$ L, Stige LC, Leirs H, Neerinckx S, Gage KL, Yang R, et al. Historical and genomic data reveal the influencing factors on global transmission velocity of plague during the third pandemic. Proc Natl Acad Sci USA 2019;116:11833-8.

13. Achtman M, Morelli G, Zhu P, Wirth T, Diehl I, Kusecek B, et al. Microevolution and history of the plague bacillus, Yersinia pestis. Proc Natl Acad Sci USA. 2004;101:17837-42.

14. Drancourt M, Roux V, Dang LV, Tran-Hung L, Castex D, Chenal-Francisque $\checkmark$, et al. Genotyping, Orientalis-like Yersinia pestis, and plague pandemics. Emerg Infect Dis. 2004;10:1585-92.

15. Drancourt M, Signoli M, Dang LV, Bizot B, Roux V, Tzortzis S, et al. Yersinia pestis Orientalis in remains of ancient plague patients. Emerg Infect Dis. 2007;13:332-3

16. Hinnebusch BJ, Perry RD, Schwan TG. Role of the Yersinia pestis hemin storage ( $h m s)$ locus in the transmission of plague by fleas. Science. 1996;273:367-70

17. Deng W, Burland V, Plunkett G 3rd, Boutin A, Mayhew GF, Liss P, et al. Genome sequence of Yersinia pestis KIM. J Bacteriol. 2002;184:4601-11.

18. Vetter SM, Eisen RJ, Schotthoefer AM, Montenieri JA, Holmes JL, Bobrov $A G$, et al. Biofilm formation is not required for early-phase transmission of Yersinia pestis. Microbiology. 2010;156:2216-25.

19. Harimalala M, Telfer S, Delatte H, Watts PC, Miarinjara A, Ramihangihajason TR, et al. Genetic structure and gene flow of the flea Xenopsylla cheopis in Madagascar and Mayotte. Parasit Vectors. 2017;10:347.

20. Eskey CR, Haas VH, Good NE. Plague in the western part of the United States. Washington: U.S. Government Printing Office; 1940.

21. Burroughs AL. Sylvatic plague studies: the vector efficiency of nine species of fleas compared with Xenopsylla cheopis. J Hyg. 1947;45:371-96.

22. Engelthaler DM, Hinnebusch BJ, Rittner CM, Gage KL. Quantitative competitive PCR as a technique for exploring flea-Yersina pestis dynamics. Am J Trop Med Hyg. 2000;62:552-60.

23. Weger-Lucarelli J, Ruckert C, Chotiwan N, Nguyen C, Garcia Luna SM, Fauver JR, et al. Vector competence of American mosquitoes for three strains of Zika virus. PLoS Negl Trop Dis. 2016;10:e0005101.

24. Flannery J, Sanz-Bernardo B, Ashby M, Brown H, Carpenter S, Cooke L, et al. Evidence of reduced viremia, pathogenicity and vector competence in a re-emerging European strain of bluetongue virus serotype 8 in sheep. Transbound Emerg Dis. 2019;66:1177-85.

25. Esteves MB, Kleina HT, Sales TM, Oliveira TP, de Lara IAR, Almeida RPP, et al. Transmission efficiency of Xylella fastidiosa subsp. pauca sequence types by sharpshooter vectors after in vitro acquisition. Phytopathology. 2019;109:286-93.

26. Link VB. A history of plague in United States of America. Public Health Monogr. 1955;26:1-120.

27. Pollitzer R. Plague. Geneva: World Health Organization;1954. https://apps. who.int/iris/handle/10665/41628

28. Chain PS, Hu P, Malfatti SA, Radnedge L, Larimer F, Vergez LM, et al. Complete genome sequence of Yersinia pestis strains Antiqua and Nepal516: evidence of gene reduction in an emerging pathogen. J Bacteriol. 2006;188:4453-63.

29. Doll JM, Zeitz PS, Ettestad P, Bucholtz AL, Davis T, Gage K. Cat-transmitted fatal pneumonic plague in a person who traveled from Colorado to Arizona. Am J Trop Med Hyg. 1994;51:109-14.

30. Sha J, Rosenzweig JA, Kirtley ML, van Lier CJ, Fitts EC, Kozlova EV, et al. A non-invasive in vivo imaging system to study dissemination of bioluminescent Yersinia pestis CO92 in a mouse model of pneumonic plague. Microb Pathog. 2013;55:39-50.

31. Lathem WW, Crosby SD, Miller VL, Goldman WE. Progression of primary pneumonic plague: a mouse model of infection, pathology, and bacterial transcriptional activity. Proc Natl Acad Sci USA. 2005:102:17786-91.

32. Willias SP, Chauhan S, Motin VL. Functional characterization of Yersinia pestis aerobic glycerol metabolism. Microb Pathog. 2014;76:33-43.

33. Choi KH, Gaynor JB, White KG, Lopez C, Bosio CM, Karkhoff-Schweizer RR, et al. A Tn7-based broad-range bacterial cloning and expression system. Nature Methods. 2005:2:443-8. 
34. Knodler LA, Crowley SM, Sham HP, Yang H, Wrande M, Ma C, et al. Noncanonical inflammasome activation of caspase-4/caspase-11 mediates epithelial defenses against enteric bacterial pathogens. Cell Host Microbe. 2014;16:249-56.

35. Datsenko KA, Wanner BL. One-step inactivation of chromosomal genes in Escherichia coli K-12 using PCR products. Proc Natl Acad Sci USA. 2000;97:6640-5

36. Derbise A, Lesic B, Dacheux D, Ghigo JM, Carniel E. A rapid and simple method for inactivating chromosomal genes in Yersinia. FEMS Immunol Med Microbiol. 2003;38:113-6.

37. Vadyvaloo V, Hinz AK. A LysR-Type Transcriptional regulator, RovM, senses nutritional cues suggesting that it is involved in metabolic adaptation of Yersinia pestis to the flea gut. PLoS ONE. 2015;10:e0137508.

38. Lorange EA, Race BL, Sebbane F, Hinnebusch BJ. Poor vector competence of fleas and the evolution of hypervirulence in Yersinia pestis. J Infect Dis. 2005;191:1907-12.

39. Lemon A, Silva-Rohwer A, Sagawa J, Vadyvaloo V. Co-infection assay to determine Yersinia pestis competitive fitness in fleas. Methods Mol Biol. 2019;2010:153-66.

40. Hinnebusch BJ, Bland DM, Bosio CF, Jarrett CO. Comparative Ability of Oropsylla montana and Xenopsylla cheopis fleas to transmit Yersinia pestis by two different mechanisms. PLoS Negl Trop Dis. 2017;11:e0005276.

41. Ren GX, Guo XP, Sun YC. HmsC Controls Yersinia pestis biofilm formation in response to redox environment. Front Cell Infect Microbiol. 2017;7:355.

42. Ren GX, Fan S, Guo XP, Chen S, Sun YC. Differential regulation of c-di-GMP metabolic enzymes by environmental signals modulates biofilm formation in Yersinia pestis. Front Microbiol. 2016;7:821.

43. Petrova OE, Sauer K. Sticky situations: key components that control bacterial surface attachment. J Bacteriol. 2012;194:2413-25.
44. Rebeil R, Jarrett CO, Driver JD, Ernst RK, Oyston PC, Hinnebusch BJ. Induction of the Yersinia pestis PhoP-PhoQ regulatory system in the flea and its role in producing a transmissible infection. J Bacteriol. 2013;195:1920-30.

45. Carniel E, Hinnebusch BJ, Carniel E, Hinnebusch BJ. Yersinia: systems biology and control. Norfolk: Caister Academic Press; 2012.

46. Jarrett CO, Deak E, Isherwood KE, Oyston PC, Fischer ER, Whitney AR, et al. Transmission of Yersinia pestis from an infectious biofilm in the flea vector. J Infect Dis. 2004;190:783-92.

47. Bobrov AG, Kirillina O, Perry RD. The phosphodiesterase activity of the HmsP EAL domain is required for negative regulation of biofilm formation in Yersinia pestis. FEMS Microbiol Lett. 2005;247:123-30.

48. Bland DM, Hinnebusch BJ. Feeding behavior modulates biofilm-mediated transmission of Yersinia pestis by the cat flea, Ctenocephalides felis. PLoS Negl Trop Dis. 2016;10:e0004413.

49. Bland DM, Jarrett CO, Bosio CF, Hinnebusch BJ. Infectious blood source alters early foregut infection and regurgitative transmission of Yersinia pestis by rodent fleas. PLoS Pathog. 2018;14:e1006859.

50. Anisimov AP, Lindler LE, Pier GB. Intraspecific diversity of Yersinia pestis. Clin Microbiol Rev. 2004;17:434-64.

51. Bellows LE, Koestler BJ, Karaba SM, Waters CM, Lathem WW. Hfq-dependent, co-ordinate control of cyclic diguanylate synthesis and catabolism in the plague pathogen Yersinia pestis. Mol Microbiol. 2012;86:661-74.

\section{Publisher's Note}

Springer Nature remains neutral with regard to jurisdictional claims in published maps and institutional affiliations.
Ready to submit your research? Choose BMC and benefit from:

- fast, convenient online submission

- thorough peer review by experienced researchers in your field

- rapid publication on acceptance

- support for research data, including large and complex data types

- gold Open Access which fosters wider collaboration and increased citations

- maximum visibility for your research: over 100M website views per year

At BMC, research is always in progress.

Learn more biomedcentral.com/submissions 